\title{
Reverse super edge-trimagic labeling for star related graphs
}

\author{
K. Amuthavalli ${ }^{1 *}$ and P. Sugapriya ${ }^{2}$
}

\begin{abstract}
A reverse edge-trimagic labeling on a graph with $p$ vertices and $q$ edges is a one-to-one map taking the vertices and edges onto the integers $1,2, \ldots, p+q$ with the property that satisfies for every edge $e$, the sum of all vertex labels incident on edge $e$ is subtracted from edge label $f(e)$ is either a constant $k_{1}$ or $k_{2}$ or $k_{3}$. The reverse edge -trimagic labeling is said to be reverse super edge - trimagic labeling if $f(v)=\{1,2, \ldots, p\}$ and $f(e)=\{p+1, p+2, \ldots, p+q\}$. In this paper, we investigate the reverse super edge -trimagic labeling of barycentric subdivision of Bi star, Degree Splitting graph of $K_{1, n} \wedge K_{1, n}$ and $K_{1, n} \cup K_{1, n}$, Corona product of $P_{3} \square K_{1}$, splitting graph of star.
\end{abstract}

\section{Keywords}

Degree splitting graph, splitting graph, Subdivision of Bi star graph, Reverse super edge-trimagic labeling.

\section{AMS Subject Classification}

$05 \mathrm{C} 78$

${ }^{1}$ Department of Mathematics, Government Arts and Science College, Veppanthattai-621116, Perambalur, Tamil Nadu, India.

2 Department of Mathematics, Sree Saraswathi Thyagaraja College, Pollachi-642107, Tamil Nadu, India.

*Corresponding author: ${ }^{1}$ thrcka@gmail.com; ${ }^{2}$ sugapriya.mat10@gmail.com

Article History: Received 24 March 2019; Accepted 17 July 2019

\section{Contents}

1 Introduction ................................. 519

2 Definition .................................... 519

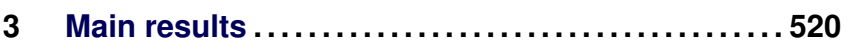

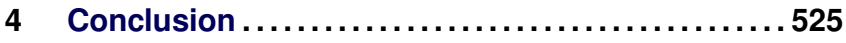

References ............................... 525

\section{Introduction}

All graphs in this paper are finite, simple and undirected. Labeling of a graph is a mapping from a set of vertices, edges or both subject to certain conditions. Motivated by magic square notation in number theory, Sedlacek [8] introduced the magic type labeling. Kotzing and Rosa [7] defined edge magic labeling in 1970.

In 2004, J.Baskar Babujee [3] introduced edge - bi magic labeling. In 2013, Jayasekaran et al.[5] introduced edge - bi magic labeling. The concept of reverse edge - magic labeling and reverse super edge -magic labeling was introduced by S. Sharif Basha [9].

Motivated by these notions, we have introduced the concept of reverse super edge - bi magic labeling in $[1,2]$. Now we extend our idea to introduced the concept of reverse super edge - trimagic labeling.

In this paper we investigate reverse super edge - trimagic labeling of barycentric subdivision of bi star, degree splitting graph of $K_{1, n} \wedge K_{1, n}$ and $K_{1, n} \cup K_{1, n}$, Corona product of $P_{3} \square K_{1}$, splitting graph of star.

\section{Definition}

Definition 2.1. [4] Let $G=(V, E)$ be a graph. Let $e=u v$ be an edge of $G$ and $w$ is not a vertex of $G$. The edge $e$ is subdivided when it is replaced by the edge $e^{\prime}=u w$ and $e^{\prime \prime}=w v$.

Definition 2.2. [4] Let $G=(V, E)$ be a graph. If every edge of graph $G$ is subdivided then the resulting graph is called barycentric subdivision of graph $G$. In other words barycentric subdivision is the graph obtained by inserting a vertex of degree 2 into every edge of original graph. The barycentric subdivision of any graph $G$ is denoted by $S^{\prime}(G)$.

Definition 2.3. [6] Let $G=(V, E)$ be a graph with $V=$ $S_{1} \cup S_{2} \cup S_{3} \cup \ldots \cup S_{t} \cup T$ where each $S_{i}$ is a set of vertices having atleast two vertices of the same degree and $T=V \bigcup_{i=1}^{t} S_{i}$. 
The degree splitting graph of $G$ denoted by $D S(G)$ is obtained from $G$ by adding vertices $u_{1}, u_{2}, \ldots, u_{t}$ and joining to each vertex of $S_{i}$ for $1 \leq i \leq t$.

Definition 2.4. A wedge is defined as an edge connecting two components of a graph, denoted as $\wedge, \omega(G \wedge)<\omega(G)$. $K_{1, m} \cup K_{1, n}$ is a two star and is a two component or a disconnected graph, whereas $K_{1, m} \wedge K_{1, n}$ is a two star but a connected graph. Which means adding a wedge to a disconnected graph with two components becomes a connected or a single component graph. And a disconnected graph with three components and two wedges becomes a connected or a single component graph.

Note: In this paper wedge is considered to connect the non pendent vertices

Definition 2.5. The splitting graph of a graph $G$ is obtained by adding to each vertex $v$ a new vertex $v$ is adjacent to every vertex that is adjacent to $v$ in $G($ i.e. $) N(v)=N\left(v^{\prime}\right)$. The resultant graph is denoted by $S^{\prime}(G)$.

Definition 2.6. The Corona $G_{1} \square G_{2}$ of two graphs $G_{1}$ and $G_{2}$ is defined as the graph $G$ obtained by taking one copy of $G_{1}$ (which has $P_{1}$ vertices) and $P_{1}$ copies of $G_{2}$ and then joining $i^{\text {th }}$ vertex of $G_{1}$ to every vertices in the $i^{\text {th }}$ copy of $G_{2}$.

\section{Main results}

Theorem 3.1. Barycentric subdivision of Bi star $S\left[B_{n, n}\right]$ is a reverse super edge-tri magic graph for $n \geq 4$.

Proof. Let $B_{n, n}$ be the bistar graph.

$$
V\left(B_{n, n}\right)=\left\{u, v, u_{i}, v_{i} ; 1 \leq i \leq n\right\}
$$

Where $u_{i}$ and $v_{i}$ are pendent vertices

$$
E\left(B_{n, n}\right)=\left\{u v, u v_{i}, v v_{i} ; 1 \leq i \leq n\right\}
$$

Let $w, u_{i}^{\prime}, v_{i}^{\prime} ; 1 \leq i \leq n$ be the newly added vertices to obtain $S\left(B_{n, n}\right)$ where $w$ is added between $u$ and $v, u_{i}^{\prime}$ is added between $u$ and $u_{i}$ for $1 \leq i \leq n$ and $v_{i}^{\prime}$ is added between $v$ and $v_{i}$ for $1 \leq i \leq n$

$$
\begin{aligned}
& \left|V\left(B_{n, n}\right)\right|=4 n+3 \\
& \left|E\left(B_{n, n}\right)\right|=4 n+2
\end{aligned}
$$

Define $f: V \bigcup E \rightarrow\{1,2, \ldots, 8 n+5\}$ as follows

The vertex labels are defined by

$$
\begin{aligned}
f(u) & =2 \\
f(w) & =1 \\
f(v) & =3
\end{aligned}
$$

For $1 \leq i \leq n$,

$$
\begin{aligned}
& f\left(u_{i}^{\prime}\right)=3+i \\
& f\left(v_{i}^{\prime}\right)=3+n-i
\end{aligned}
$$

For $1 \leq i \leq n-2$,

$$
\begin{aligned}
f\left(u_{i}\right) & =4 n-2 i+2 \\
f\left(v_{i}\right) & =4 n-2 i+5 \\
f\left(u_{n-1}\right) & =4 n+2 \\
f\left(u_{n}\right) & =2 n+4
\end{aligned}
$$

The edge labels are defined by

$$
\begin{aligned}
& f(u w)=4 n+4 \\
& f(v w)=4 n+5
\end{aligned}
$$

For $1 \leq i \leq n$,

$$
\begin{gathered}
f\left(u u_{i}^{\prime}\right)=4 n+6+i \\
f\left(v v_{i}^{\prime}\right)=5 n+7+i \\
f\left(v_{i} v_{i}^{\prime}\right)=7 n+8-i
\end{gathered}
$$

For $1 \leq i \leq n-3$,

$$
f\left(u_{i} u_{i}^{\prime}\right)=8 n+6-i
$$

For $-2 \leq i \leq n-1$,

$$
\begin{aligned}
& f\left(u_{i} u_{i}^{\prime}\right)=n^{2}+4 n-n i-i+5 \\
& f\left(u_{n} u_{n}^{\prime}\right)=7 n+8
\end{aligned}
$$

Then the constants $k_{1}, k_{2}, k_{3}$ of reverse super edge- trimagic are obtained as follows.

To find $k_{1}$ :

$$
\begin{aligned}
3 n k_{1}= & f(u v)-[f(u)+f(w)] \\
& +f(v w)-[f(v)+f(w)] \\
& +\sum_{i=1}^{n}\left[f\left(u u_{i}^{\prime}\right)-\left[f(u)+f\left(u_{i}^{\prime}\right)\right]\right] \\
& +\sum_{i=1}^{n}\left[f\left(v v_{i}^{\prime}\right)-\left[f(v)+f\left(v_{i}^{\prime}\right)\right]\right] \\
& +\sum_{i=1}^{n-3}\left[f\left(u_{i}^{\prime} u_{i}\right)-\left[f\left(u_{i}^{\prime}\right)+f\left(u_{i}\right)\right]\right] \\
& +f\left(u_{n}^{\prime} u_{n}\right)-\left[f\left(u_{n}^{\prime} u_{n}\right)\right] \\
3 n k_{1}= & \{(4 n+4)-(2+1)\}+\{(4 n+5)-(3+1)\} \\
& +\sum_{i=1}^{n}[(5 n+7+i)-(3+3+n+i)] \\
& +\sum_{i=1}^{n-3}[(8 n-i+6)-(3+i+4 n-2 i+2)] \\
& +[(7 n+3)-(3+n+2 n+4)] \\
k_{1}= & 4 n+1
\end{aligned}
$$


To find $k_{2}$ :

$$
\begin{aligned}
(n+1) k_{2}= & \sum_{i=1}^{n}\left[f\left(v v_{i}^{\prime}\right)-\left[f(v)+f\left(v_{i}^{\prime}\right)\right]\right] \\
& +f\left(u_{n-2}^{\prime} u_{n-2}\right)-\left[f\left(u_{n-2}^{\prime}\right)+f\left(u_{n-2}\right)\right] \\
= & \sum_{i=1}^{n}(7 n+8+i) \\
& -[(4 n-2 i+5)+(3+n+i)] \\
& +\left[\left\{n^{2}+4 n-n(n-2)-(n-2)+5\right\}\right. \\
& +\{(3+(n-2))+(4 n-2(n-2)+2)\}] \\
K_{2}= & 2 n
\end{aligned}
$$

To find $k_{3}$ :

$$
\begin{aligned}
k_{3}= & f\left(u_{n-1}^{\prime} u_{n-1}\right)-\left[f\left(u_{n-1}^{\prime}\right)+f\left(u_{n-1}\right)\right] \\
k_{3}= & \left\{n^{2}+4 n-n(n-1)-(n-1)+5\right\} \\
& +\{(3+(n-1))+(4 n-2)\} \\
k_{3}= & 2-n
\end{aligned}
$$

Thus $f$ is a reverse edge-trimagic labeling.

Hence, $S\left(B_{n, n}\right)$ is a reverse super edge- trimagic graph.

\section{Illustration 3.1}

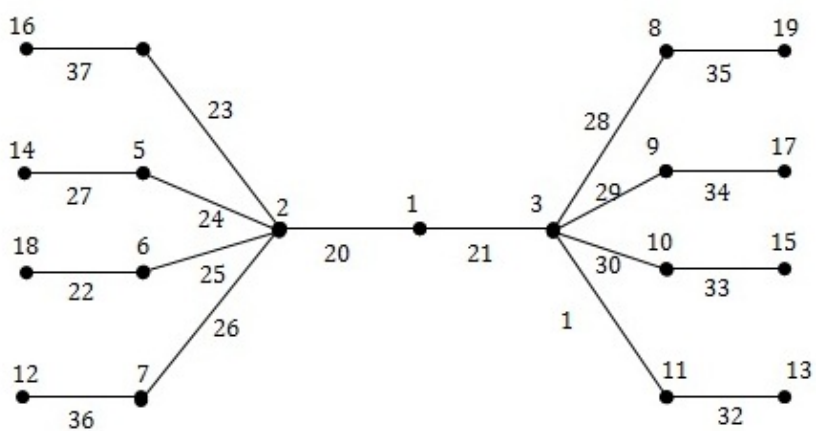

Fig 3.1: $S\left(B_{4,4}\right)$ is a Reverse Super edge - trimagic graph.

Theorem 3.2. The degree splitting graph

$$
D S\left[K_{1, n} \bigcup K_{1, n}\right]
$$

is a reverse super edge-trimagic graph for $n \geq 2$.

Proof. Let

$$
V\left[K_{1, n} \bigcup K_{1, n}\right]=\left\{u, u_{i}, v, v_{i} ; 1 \leq i \leq n\right\}
$$

where $u_{i}$ 's and $v_{i}$ 's are pendent vertices.

$$
V\left[K_{1, n} \bigcup K_{1, n}\right]=V_{1} \bigcup V_{2}
$$

where $V_{1}=\{u, v\}$

$$
V_{2}=\left\{u_{i}, v_{i} ; 1 \leq i \leq n\right\}
$$

Now, in order to obtain $D S\left[K_{1, n} \bigcup K_{1, n}\right]$ from $K_{1, n} \cup K_{1, n}$ we add $w_{1}$ and $w_{2}$ corresponding to $V_{1}$ and $V_{2}$. Then

$$
\begin{aligned}
V\left[D S\left[K_{1, n} \bigcup K_{1, n}\right]\right]= & \left\{u, u_{i}, v, v_{i}, w_{1}, w_{2} ; 1 \leq i \leq n\right\} \\
W\left[D S\left[K_{1, n} \bigcup K_{1, n}\right]\right]= & \left\{u w_{1}, u_{i} w_{2}, v w_{1}, u u_{i}, v v_{i}, v_{i} w_{2}\right\} \\
& 1 \leq i \leq n
\end{aligned}
$$

We note that

$$
\begin{aligned}
& \left|V\left[D S\left[K_{1, n} \bigcup K_{1, n}\right]\right]\right|=2 n+4 \\
& \left|W\left[D S\left[K_{1, n} \bigcup K_{1, n}\right]\right]\right|=4 n+2
\end{aligned}
$$

Define $f: V \bigcup W \rightarrow\{1,2, \ldots, 6 n+6\}$ as follows

The vertex labels are defined by

$$
\begin{aligned}
f(u) & =2 \\
f(v) & =3 \\
f\left(w_{1}\right) & =4 \\
f\left(w_{2}\right) & =1
\end{aligned}
$$

For $1 \leq i \leq n$,

$$
\begin{aligned}
& f\left(u_{i}^{\prime}\right)=4+i \\
& f\left(v_{i}^{\prime}\right)=4+n+i
\end{aligned}
$$

The edge labels are defined as follows For $1 \leq i \leq n$,

$$
\begin{aligned}
f\left(u_{i} w_{2}\right) & =2 n+4+i \\
f\left(v_{i} w_{2}\right) & =3 n+4+i \\
f\left(u u_{i}\right) & =4 n+6+i \\
f\left(v v_{i}\right) & =5 n+6+i \\
f\left(u w_{1}\right) & =4 n+5 \\
f\left(v w_{1}\right) & =4 n+6
\end{aligned}
$$

Then the constants $k_{1}, k_{2}, k_{3}$ of reverse super edge- trimagic are obtained as follows.

To find $k_{1}$ :

$$
\begin{aligned}
(n+2) k_{1}= & {\left[f\left(u w_{1}\right)-\left\{f(u)+f\left(w_{1}\right)\right\}\right] } \\
& +\left[f\left(v w_{1}\right)-\left\{f(v)+f\left(w_{1}\right)\right\}\right] \\
& +\sum_{i=1}^{n}\left[f\left(v_{i} v\right)-\left\{f\left(v_{i}\right)+f(v)\right\}\right] \\
(n+2) k_{1}= & {[(4 n+5)-(2+4)]+[(4 n+6)-(3+4)] } \\
& +\sum_{i=1}^{n}[(5 n+6+i)-(4+n+3+i)] \\
k_{1}= & 4 n-1
\end{aligned}
$$


To find $k_{2}$ :

$$
\begin{aligned}
n k_{2} & =\sum_{i=1}^{n}\left[f\left(u u_{i}^{\prime}\right)-\left\{f(u)+f\left(u_{i}\right)\right\}\right] \\
& =\sum_{i=1}^{n}[4 n+6+i-\{2+4+i\}] \\
K_{2} & =4 n
\end{aligned}
$$

\section{To find $k_{3}$ :}

$$
\begin{aligned}
2 n k_{3}= & \sum_{i=1}^{n}\left[f\left(u_{i} w_{2}\right)-\left\{f\left(u_{i}\right)+f\left(w_{2}\right)\right\}\right] \\
& +\sum_{i=1}^{n}\left[f\left(v_{i} w_{2}\right)-\left\{f\left(v_{i}\right)+f\left(w_{2}\right)\right\}\right] \\
= & \sum_{i=1}^{n}[(2 n+4+i)-\{4+i+1\}] \\
& +\sum_{i=1}^{n}[(3 n+4+i)-\{4+n+i+1\}] \\
k_{3}= & 2 n-1
\end{aligned}
$$

Thus $f$ is a reverse super edge-trimagic labeling.

Hence $D S\left[K_{1, n} \cup K_{1, n}\right]$ is a reverse super edge- trimagic graph for $n \geq 2$.

\section{Illustration 3.2}

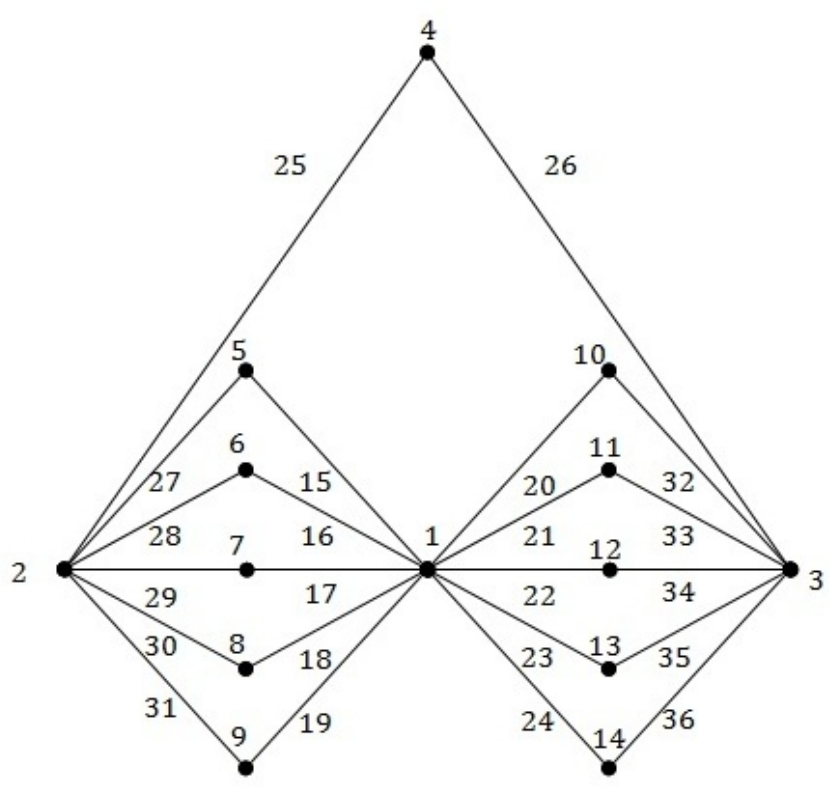

Fig 3.2: $D S\left[K_{1,5} \cup K_{1,5}\right]$ is a Reverse Super Edge-Trimagic Graph

Theorem 3.3. The degree splitting graph

$$
D S\left[K_{1, n} \wedge K_{1, n}\right]
$$

is a reverse super edge-trimagic graph for $n \geq 2$.
Proof. Let

$$
V\left[K_{1, n} \wedge K_{1, n}\right]=\left\{u, u_{i}, v, v_{i} ; 1 \leq i \leq n\right\}
$$

where $u_{i}$ 's and $v_{i}$ 's are pendent vertices.

$$
V\left[K_{1, n} \wedge K_{1, n}\right]=V_{1} \bigcup V_{2}
$$

where $V_{1}=\{u, v\}$

$$
V_{2}=\left\{u_{i}, v_{i} ; 1 \leq i \leq n\right\}
$$

Now, in order to obtain $D S\left[K_{1, n} \wedge K_{1, n}\right]$ from $K_{1, n} \wedge K_{1, n}$ we add $w_{1}$ and $w_{2}$ corresponding to $V_{1}$ and $V_{2}$. Then

$$
\begin{aligned}
V\left[D S\left[K_{1, n} \wedge K_{1, n}\right]\right]= & \left\{u, u_{i}, v, v_{i}, w_{1}, w_{2} ; 1 \leq i \leq n\right\} \\
W\left[D S\left[K_{1, n} \wedge K_{1, n}\right]\right]= & \left\{u v, u w_{1}, u_{i} w_{2}, v w_{1}, u u_{i}, v v_{i}, v_{i} w_{2}\right\} \\
& 1 \leq i \leq n
\end{aligned}
$$

We note that

$$
\begin{aligned}
& \left|V\left[D S\left[K_{1, n} \wedge K_{1, n}\right]\right]\right|=2 n+4 \\
& \left|W\left[D S\left[K_{1, n} \wedge K_{1, n}\right]\right]\right|=4 n+3
\end{aligned}
$$

Define $f: V \bigcup W \rightarrow\{1,2, \ldots, 6 n+6\}$ as follows

The vertex labels are defined by

$$
\begin{aligned}
f\left(w_{1}\right) & =1 \\
f(u) & =2 \\
f(v) & =3 \\
f\left(w_{2}\right) & =2 n+4
\end{aligned}
$$

For $1 \leq i \leq n$,

$$
\begin{aligned}
& f\left(u_{i}^{\prime}\right)=3+i \\
& f\left(v_{i}^{\prime}\right)=3+n+i
\end{aligned}
$$

The edge labels are defined as follows

$$
\begin{aligned}
f\left(u w_{1}\right) & =2 n+5 \\
f\left(v w_{1}\right) & =2 n+6 \\
f(u v) & =2 n+7
\end{aligned}
$$

For $1 \leq i \leq n$,

$$
\begin{aligned}
f\left(u u_{i}\right) & =4 n+7+i \\
f\left(v v_{i}\right) & =5 n+7+i \\
f\left(u_{i} w_{2}\right) & =2 n+7+i \\
f\left(v_{i} w_{2}\right) & =3 n+7+i
\end{aligned}
$$

Then the constants $k_{1}, k_{2}, k_{3}$ of reverse super edge- trimagic are obtained as follows. 
To find $k_{1}$ :

$$
\begin{aligned}
(n+3) k_{1}= & {[f(u v)-\{f(u)+f(v)\}] } \\
& +\left[f\left(u w_{1}\right)-\left\{f(u)+f\left(w_{1}\right)\right\}\right] \\
& +\left[f\left(v w_{1}\right)-\left\{f(v)+f\left(w_{1}\right)\right\}\right] \\
& +\sum_{i=1}^{n}\left[f\left(u w_{i}\right)-\left\{f(u)+f\left(u_{i}\right)\right\}\right] \\
(n+3) k_{1}= & {[(2 n+7)-(1+3)] } \\
& +[(2 n+5)-(2+1)] \\
& +[(2 n+6)-(3+1)] \\
& +\sum_{i=1}^{n}[(2 n+7+i)-(2+3+i)]
\end{aligned}
$$$$
k_{1}=2 n+2
$$

To find $k_{2}$ :

$$
\begin{aligned}
n k_{2} & =\sum_{i=1}^{n}\left[f\left(v v_{i}^{\prime}\right)-\left\{f(v)+f\left(v_{i}\right)\right\}\right] \\
& =\sum_{i=1}^{n}[3 n+7+i-\{3+3+n+i\}] \\
K_{2} & =2 n+1
\end{aligned}
$$

To find $k_{3}$ :

$$
\begin{aligned}
2 n k_{3}= & \sum_{i=1}^{n}\left[f\left(u_{i} w_{2}\right)-\left\{f\left(u_{i}\right)+f\left(w_{2}\right)\right\}\right] \\
& +\sum_{i=1}^{n}\left[f\left(v_{i} w_{2}\right)-\left\{f\left(v_{i}\right)+f\left(w_{2}\right)\right\}\right] \\
= & \sum_{i=1}^{n}[(4 n+7+i)-\{3+i++2 n+4\}] \\
& +\sum_{i=1}^{n}[(5 n+7+i)-\{3+n+i+2 n+4\}] \\
k_{3}=2 n &
\end{aligned}
$$

Thus $f$ is a reverse super edge-trimagic labeling. Hence

$$
D S\left[K_{1, n} \wedge K_{1, n}\right]
$$

is a reverse super edge- trimagic graph for $n \geq 2$.

\section{Illustration 3.3}

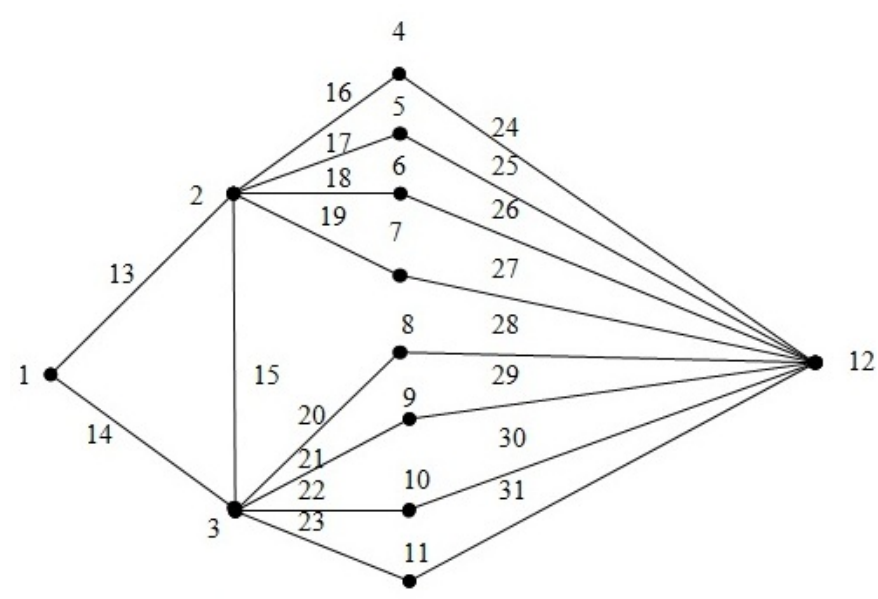

Fig 3.3: $D S\left[K_{1,4} \wedge K_{1,4}\right]$ is a Reverse Super Edge-Trimagic graph

Theorem 3.4. The graph

$$
P_{3} \square K_{1, n}
$$

is a reverse super edge-trimagic labeling for $n \geq 2$.

Proof. Let

$$
\begin{aligned}
V\left[P_{3} \square K_{1, n}\right] & =\left\{u, u_{i}, v, v_{i}, w, w_{i} ; 1 \leq i \leq n\right\} \\
W\left[P_{3} \square K_{1, n}\right] & =\left\{u v, u u_{i}, v w, v v_{i}, w w_{i} ; 1 \leq i \leq n\right\} \\
\left.\mid V\left[P_{3} \square K_{1, n}\right]\right] \mid & =3 n+3 \\
\left.\mid W\left[P_{3} \square K_{1, n}\right]\right] \mid & =3 n+2
\end{aligned}
$$

Define $f: V \bigcup W \rightarrow\{1,2, \ldots, 6 n+5\}$ as follows

The vertex labels are defined by

$$
\begin{aligned}
f(u) & =1 \\
f(v) & =2 \\
f(w) & =3
\end{aligned}
$$

For $1 \leq i \leq n$,

$$
\begin{aligned}
& f\left(u_{i}\right)=3+2 n+i \\
& f\left(v_{i}\right)=3+n+i \\
& f\left(w_{i}\right)=3+i
\end{aligned}
$$

The edge labels are defined by

$$
\begin{aligned}
& f(u v)=3 n+4 \\
& f(v w)=3 n+5
\end{aligned}
$$


For $1 \leq i \leq n$,

$$
\begin{aligned}
f\left(u u_{i}\right) & =5 n+5+i \\
f\left(v v_{i}\right) & =4 n+5+i \\
f\left(w w_{i}\right) & =3 n+5+i
\end{aligned}
$$

Then the constants $k_{1}, k_{2}, k_{3}$ of reverse super edge- trimagic labeling are obtained as follows.

To find $k_{1}$ :

$$
\begin{aligned}
(n+1) k_{1}= & {[f(u v)-\{f(u)+f(v)\}] } \\
& +\left[f\left(u u_{i}\right)-\left\{f(u)+f\left(u_{i}\right)\right\}\right] \\
(n+1) k_{1}= & {[(3 n+4)-(1+2)] } \\
& +\sum_{i=1}^{n}[(5 n+5+i)-(1+3+2 n+i)] \\
k_{1}= & 3 n+1
\end{aligned}
$$

To find $k_{2}$ :

$$
\begin{aligned}
(n+1) k_{2}= & {[f(v w)-\{f(v)+f(w)\}] } \\
& +\sum_{i=1}^{n}\left[f\left(v v_{i}\right)-\left\{f(v)+f\left(v_{i}\right)\right\}\right] \\
= & {[(3 n+5)-\{2+3\}] } \\
& +\sum_{i=1}^{n}[4 n+5+i-\{2+3+n+i\}] \\
K_{2}= & 3 n
\end{aligned}
$$

To find $k_{3}$ :

$$
\begin{aligned}
n k_{3} & =\sum_{i=1}^{n}\left[f\left(w w_{i}\right)-\left\{f(w)+f\left(w_{i}\right)\right\}\right] \\
n k_{3} & =\sum_{i=1}^{n}[(3 n+5+i)-\{3+3+i\}] \\
k_{3} & =3 n-1
\end{aligned}
$$

Thus $f$ is a reverse super edge-trimagic labeling.

Hence

$$
P_{3} \square K_{1, n}
$$

is a reverse super edge- trimagic labeling for $n \geq 2$.

\section{Illustration 3.4}

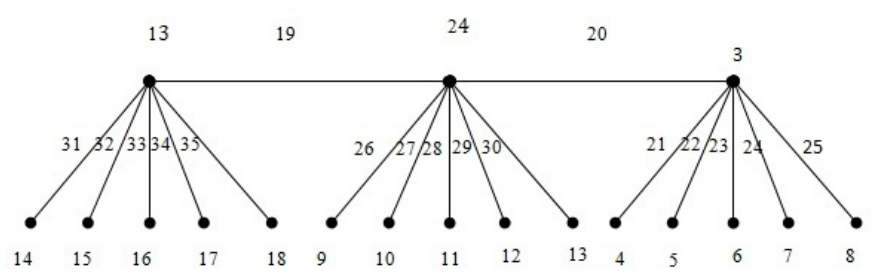

Fig 3.4: $P_{5} \square K_{1,5}$ is a Reverse Super Edge - Trimagic graph
Theorem 3.5. The Splitting graph of star graph $S^{\prime}\left(K_{1, n}\right)$ is a reverse super edge-trimagic graph for $n \geq 3$.

Proof. The splitting graph of star graph $S^{\prime}\left(K_{1, n}\right)$ is obtained by adding vertices $v$ and $v_{i}, 1 \leq i \leq n$ such that $u$ and $u_{i}, 1 \leq$ $i \leq n$ is adjacent to every vertex that is adjacent to $v$ and $v_{i}, 1 \leq i \leq n$ in $K_{1, n}$.

Let

$$
\begin{gathered}
V\left[S^{\prime}\left(K_{1, n}\right)\right]=\left\{u, u_{i}, v, v_{i} ; 1 \leq i \leq n\right\} \\
E\left[S^{\prime}\left(K_{1, n}\right)\right]=\left\{v v_{i}, v u_{i}, u v_{i} ; 1 \leq i \leq n\right\}
\end{gathered}
$$

We note that

$$
\begin{aligned}
& \left.\mid V\left[S^{\prime}\left(K_{1, n}\right)\right]\right] \mid=2 n+2 \\
& \left.\mid E\left[S^{\prime}\left(K_{1, n}\right)\right]\right] \mid=3 n
\end{aligned}
$$

Define $f: V \bigcup E \rightarrow\{1,2, \ldots, 5 n+2\}$ as follows

The vertex labels are defined by For $1 \leq i \leq n$,

$$
\begin{aligned}
f\left(u_{i}\right) & =1 \\
f\left(v_{i}\right) & =n+i \\
f(u) & =2 n+i \\
f(v) & =2 n+2
\end{aligned}
$$

The edge labels are defined by For $1 \leq i \leq n$,

$$
\begin{aligned}
& f\left(v u_{i}\right)=2 n+3+i \\
& f\left(v v_{i}\right)=3 n+3+i \\
& f\left(u v_{i}\right)=2 n+3
\end{aligned}
$$

For $2 \leq i \leq n$,

$$
f\left(u v_{i}\right)=4 n+2+i
$$

Then the constants $k_{1}, k_{2}, k_{3}$ of reverse super edge- trimagic labeling are obtained as follows.

\section{To find $k_{1}$ :}

$$
\begin{aligned}
2 n k_{1}= & \sum_{i=1}^{n}\left[f\left(v u_{i}\right)-\left\{f(v)+f\left(u_{i}\right)\right\}\right] \\
& +\sum_{i=1}^{n}\left[f\left(v v_{i}\right)-\left\{f(v)+f\left(v_{i}\right)\right\}\right] \\
2 n k_{1}= & \sum_{i=1}^{n}[(2 n+3+i)-((2 n+2)+i)] \\
& +\sum_{i=1}^{n}[(3 n+3+i)-((2 n+2)+(n+i))] \\
k_{1}= & 1
\end{aligned}
$$

To find $k_{2}$ :

$$
\begin{aligned}
(n-1) k_{2} & =\sum_{i=1}^{n}\left[f\left(u v_{i}\right)-\left\{f(u)+f\left(v_{i}\right)\right\}\right] \\
(n-1) k_{2} & =\sum_{i=1}^{n}[(4 n+2+i)-\{(2 n+1)+(n+i)\}] \\
K_{2} & =n+1
\end{aligned}
$$


To find $k_{3}$ :

$$
\begin{aligned}
& k_{3}=f\left(u v_{i}\right)-\left\{f(u)+f\left(v_{i}\right)\right\} \\
& k_{3}=(2 n+3)-\{2 n+1+n+1\} \\
& k_{3}=1-n
\end{aligned}
$$

Thus $f$ is a reverse super edge-trimagic labeling.

Hence

$$
S^{\prime}\left(K_{1, n}\right)
$$

is a reverse super edge- trimagic graph $n \geq 3$.

\section{Illustration 3.5}

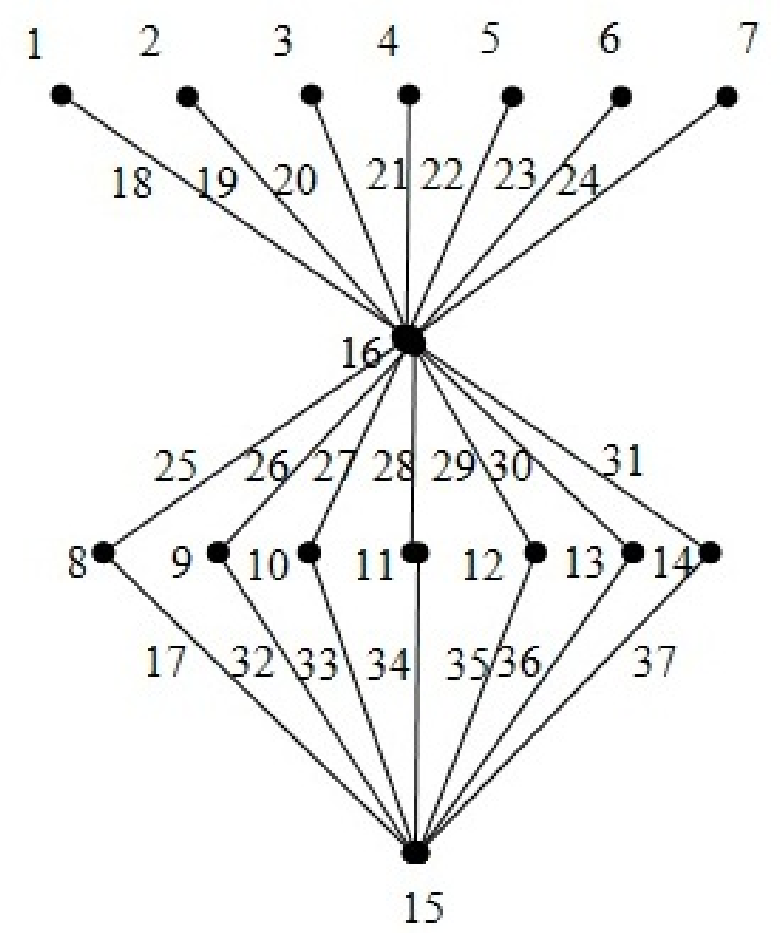

Fig 3.5: $S^{\prime}\left(K_{1,7}\right)$ is a Reverse Super Edge - Trimagic graph

\section{Conclusion}

The concept of reverse super edge - trimagic labeling of several classes of graphs are discussed here.

\section{References}

[1] K. Amuthavalli, P. Sugapriya, S. Mythili, M. Priyanga, Reverse Super Edge - Bi Magic Labeling, Mathematical Sciences International Research Journal, 7(2), (2018), 176-180.

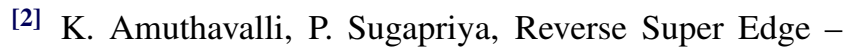
Bimagic labeling of star related graphs, International Journal for Research in Engineering Applications \& Management, 05(02)(2019), 1-12.
[3] Baskar Babujee, On Edge Bi magic labeling, Journal of combinatorics Information and System Sciences, 1(4), (2004) 239-244.

[4] M. I. Bosmia, K. K. Kanani, Divisor cordial labeling in the context of graph operations on Bistar, Global Journal of Pure and Applied Mathematics, 12(3), (2016), 26052618.

[5] C. Jayasekaran, M. Regees and C. Davidraj, Edgetrimagic labelling of some graphs, Intern.J. Combinatorial Graph theory and Applications, 6(2), (2013), 175186.

[6] J. A. Gallion, A .Dynamic Survey of Graph Labeling, Electronic J. Combinations, 19, (2016), DS6.

[7] A. Kotizing and A. Rosa, Magic Valuations of finite graphs, Canad. Math. Bull, 13, (1970), 451-461.

[8] J. Sedlacek, Problem 27 : In theory of graphs and its applications, Proc. Symposium Smolenice, (1963), 163167.

[9] S. Sharief Basha, Reverse super edge-magic labeling on $\mathrm{W}$-trees, International Journal of Computer Engineering in Research Trends, 2(11)(2015), 719-721

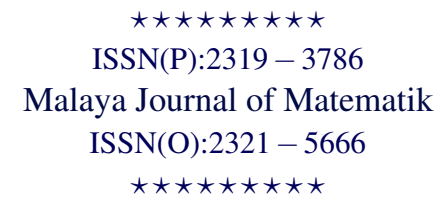

